\title{
Body Image, Physical Activity and Sport Involvement: A Study on Gender Differences
}
Authors' contribution:
A) conception and design of the study
B) acquisition of data
C) analysis and interpretation of data
D) manuscript preparation
E) obtaining funding

\author{
Ho Keat Leng ${ }^{\text {A-E }}$, Yi Xian Philip Phua ${ }^{\text {B,D }}$, Yifan Yang ${ }^{\text {C,D }}$ \\ Nanyang Technological University, Singapore
}

ABSTRACT

The aim of this study is to examine whether sport involvement i.e. identification with the domain of sports affect satisfaction with body image in men and women. 158 respondents from a tertiary educational institution provided data on their satisfaction with their body image, level of sport involvement, and level of physical activity. Independent t-tests were conducted to compare differences between and within genders. Female respondents $(n=40)$ who were high in sport involvement reported a significantly lower level of dissatisfaction with their body image $(\mathrm{M}=.55, \mathrm{SD}=.78)$ when compared to female respondents $(\mathrm{n}=44)$ who were low in sport involvement $(\mathrm{M}=.84, \mathrm{SD}=.83$; $\mathrm{t}=1.65, \mathrm{p}<.05)$. There was no significant difference amongst male respondents. There was also no significant difference in satisfaction with body image across groups with varying levels of physical activity. This suggests that being involved in sport can affect body image satisfaction amongst females.

KEYWORDS $\quad$ body image satisfaction, sport involvement, gender differences

\section{Introduction}

There are many studies on body image satisfaction. This is because it is an important area as dissatisfaction with body image can result in unhealthy behaviours including maladaptive eating and exercise patterns (Anton, Perri, \& Riley III, 2000; Grogan, 2006). While body image satisfaction can affect both genders, there is a larger number of studies focused on body image satisfaction among females. Studies that have examined body image satisfaction for both genders have found that there is a difference across genders. In addition, while some studies have found that body image satisfaction is correlated with the level of exercise and physical activity, others have suggested this relationship can be complex.

Body image satisfaction is in the cognitive domain. As such, this study aims to examine whether a cognitive construct like sport involvement can better explain the effect on body image satisfaction compared to a behavioural construct like physical activity. In addition, the study will also examine differences in body image satisfaction between the genders.

\section{Literature review}

Body image is defined as the internal representation of a person's outer appearance (Thompson, Heinberg, 
Altabe, \& Tantleff-Dunn, 1999). It relates to a person's perceptions, feelings and thoughts about the body and incorporates body size estimation, evaluation of body attractiveness and emotions associated with body size (Grogan, 2006). While women tend to prefer a smaller and more petite body, men can either prefer a larger or smaller body size (Cohane \& Pope Jr, 2001; Gaspar, Amaral, Oliveira, \& Borges, 2011; Ingledew \& Sullivan, 2002; Smith, Wright, Ross, \& Warmington, 2006). Specifically, body image satisfaction amongst men is associated with lower body fat (MacKinnon et al., 2003). Hence, men can prefer a larger body size if the increase is due to a leaner and more muscular body shape.

When there is a discrepancy between an individual's perceived body image with an ideal body image, it will result in body image dissatisfaction. This phenomenon has been found to be a key risk factor for several health issues including eating disorders and avoidance of physical activity (Anton et al., 2000; Carmona, TorneroQuinones, \& Sierra-Robles, 2015; Černelič-Bizjak \& Jenko-Pražnikar, 2014; Grogan, 2006; Pila, Barlow, Wrosch, \& Sabiston, 2016; You \& Shin, 2016). Body image dissatisfaction is more prevalent among females, but can affect males especially for those who are under 18 years of age (Anton et al., 2000; Cohane \& Pope Jr, 2001; Gaspar et al., 2011; Perelman, Buscemi, Dougherty, \& Haedt-Matt, 2018; Posavac, Posavac, \& Weigel, 2001; Thompson \& Stice, 2001).

Some researchers have also suggested that there are cultural and ethnic differences in body image dissatisfaction. For example, Asian British women were found to have lower body image dissatisfaction compared to their western counterparts. This was attributed to Asian women being inherently slimmer than white women (Altabe, 1998; Wardle, Bindra, Fairclough, \& Westcombe, 1993).

However, Haworth-Hoeppner (2000) suggested that cultural influence could be mediated through interactions with members of social groups, particularly with family members. This is because the most common source of health information is often from parents (Nustad, Adams, \& Moore, 2008). As such, sociocultural theory can explain body image dissatisfaction as resulting from the social pressures faced by an individual to conform to specific standards.

The concept of body image is thus malleable and there are many sources of influence including family and friends, traditional media and social media. These sources of influence can affect societal members on acceptable body images and sizes (Baker, Ferszt, \& Breines, 2019; Diedrichs \& Lee, 2011; Graham, Eich, Kephart, \& Peterson, 2000; Griffiths, Murray, Krug, \& McLean, 2018; Lewallen \& Behm-Morawitz, 2016; Meng, Bissell, \& Pan, 2015; Nerini, 2015). Of greater concern, they can also cause body image dissatisfaction by influencing individuals to internalise an ideal body size that is unrealistic (Baker et al., 2019; Swami, Steadman, \& Tovée, 2009; Thompson et al., 1999; Thompson \& Stice, 2001).

In order to reduce body image dissatisfaction, there is some evidence that engaging in physical activity or exercise can be effective (Fisher \& Thompson, 1994; Gaspar et al., 2011; SantaBarbara, Whitworth, \& Ciccolo, 2017). In a meta-analysis conducted by Hausenblas and Fallon (2006), females who engage in exercise were found to have improved self-esteem and lower dissatisfaction with their body image. Some characteristics of exercise e.g. duration and type did not moderate the effect size of body image dissatisfaction, while others had a significant moderating effect e.g. frequency and specificity (Campbell \& Hausenblas, 2009).

However, the effect of physical activity on body image satisfaction is not consistent across studies (Vocks, Hechler, Rohrig, \& Legenbauer, 2009). Some studies suggest that exercise behaviour can sometimes increase body image dissatisfaction. Prichard and Tiggemann (2008) found that exercising in fitness centres was associated with a negative body image. They suggest that the exercise environment in fitness centres can promote an unrealistic ideal body image through mirrors, posters and other users in tight and revealing clothing. Consequently, individuals who are exercising in fitness centres are more likely to feel dissatisfied with their body image compared to individuals exercising in other venues (Prichard \& Tiggemann, 2008). 
As athletes are more likely to engage in high levels of physical activity, some researchers have attempted to examine the relationship between physical activity and body image by focusing on athletes. A meta-analysis showed that athletes in general had a more positive body image when compared to non-athletes although the effect size is small (Hausenblas \& Downs, 2001). However, other studies have noted that although athletes engage in high levels of physical activity, they can also report body image dissatisfaction depending on the type of sport (Abbott \& Barber, 2011; Hulley \& Hill, 2001; Kantanista et al., 2018; Nerini, 2015; Swami et al., 2009). As such, it is evident that the relationship between physical activity and body image satisfaction is neither simple nor direct.

It has been suggested that besides external sources of influence, the effect of physical activity on body image satisfaction may depend on the individual's motivation for exercising and internalisation of ideal body size (Homan \& Tylka, 2014; Swami et al., 2009). Raedeke (2007) found that while exercise was associated with enhanced positive affect, individuals who enjoyed the exercise had the greatest increase in positive affect. In addition, studies have shown that women who exercise primarily for appearance-related reasons are more likely to exhibit symptoms of eating disorder, as well as lower self-esteem (Gonçalves \& Gomes, 2012; Strelan, Mehaffey, \& Tiggemann, 2003).

Other studies have suggested that even when participating in physical activity can reduce body image dissatisfaction, it may be challenging to do so. This is because females who are less satisfied with their body image are also less likely to participate in physical activity (Pila et al., 2016). This was attributed to an abandonment of healthy living principles like proper eating and exercise as a consequence of being unable to achieve a desired body shape (Anton et al., 2000).

Body image satisfaction is a psychological construct. Hence, instead of examining a behavioural construct like physical activity, it might be more illuminative to examine the relationship between body image satisfaction with another psychological construct (Prichard \& Tiggemann, 2008). One possible construct that has yet to be examined in the literature in relation to body image dissatisfaction is that of sport involvement.

Sport involvement is defined as the love of, and bond with sports. It refers to the extent of personal relevance of sport to an individual (Gwinner \& Swanson, 2003). Hence, sport involvement is the cognitive affiliation towards sports and is distinct from behavioural measures such as the sport participation rate. Earlier studies have found that sport involvement can affect sports consumption behaviour (Chew \& Leng, 2014). In addition, given the gendered nature of sport, the level of sport involvement is generally higher among males (Leng, Kuo, Baysa-Pee, \& Tay, 2014).

However, sport involvement is distinct from exercise behaviour or intention. Abbott and Barber (2011) have found that there is a difference between participating in physical activity and a more structured activity like sports. In addition, an individual may be highly involved in sport but exhibit no exercise behaviour nor behavioural intention. This is because sport involvement can include passive activities like watching and reading about sports. As such, using sport involvement as a variable in a study on body image satisfaction may provide a different perspective from earlier studies.

The above shows that the study of body image satisfaction is an important topic. As females are more likely to be associated with body image dissatisfaction, there is a larger number of studies focused on this demographic segment. Studies that have compared body image satisfaction between the genders have generally found that females prefer a smaller body size while males can prefer either a larger or smaller body size. While studies have found some evidence that exercise or physical activity can reduce dissatisfaction with body image, the relationship between the two is complex. Hence, the aim of this study is to examine whether a psychological construct like sport involvement can provide a different perspective on reducing body image dissatisfaction. 


\section{Methods}

\section{Procedure}

220 respondents from a tertiary educational institution were recruited for this study. Respondents were asked to complete a survey instrument which captured data on their satisfaction with their body image, level of sport involvement, and level of physical activity. The data was then analysed using statistical software.

\section{Measures}

Body image satisfaction is commonly measured using silhouette scales. However, there are limitations associated with the use of such scales including scale coarseness due to the small number of images in the scale and changes in body size across the scale in non-equal intervals (Gardner, Stark, Jackson, \& Friedman, 1999). Attempts to address these limitations resulted in the development of several different scales that can be used to measure body size perception (Gardner et al., 1999).

In this study, Stunkard's body image scale was selected as a measure as it had been used in many other studies. More importantly, its validity as a measure of body size perception has been demonstrated as there is a significant correlation between the images selected with respondents' actual weight and Body Mass Index. It was also found that the validity of Stunkard's body image scale is comparable to several other measures (Gardner et al., 1999; Stunkard, 2000). Hence, this suggests that it is an appropriate measure for this study.

The Stunkard scale consists of nine male and female schematic figures ranging from very thin to very fat. Respondents were asked to select the figure that most closely represent their body and the figure that represents their ideal body image. The difference between the two represents the measure of body image dissatisfaction.

Sport involvement was measured using a 3-item, 7-point Likert scale adapted from the instrument used by Gwinner and Swanson to assess domain involvement. In an earlier study, the sport involvement scale was reported to have good internal consistency with a Cronbach alpha coefficient of 0.87 (Gwinner \& Swanson, 2003). In this study, the Cronbach alpha coefficient was 0.80 which is considered acceptable. The items are as follow.

a) Sports is very important to me.

b) I think about sports all of the time.

c) I watch sports whenever I can.

The level of physical activity was measured by the short-form of the International Physical Activity Questionnaire (IPAQ) used in many studies (Craig et al., 2003). This measures the frequency and duration of physical activity of respondents in the last 7 days and converts it to multiples of the resting metabolic rate (MET). In the IPAQ, walking is given a value of 3.3 METs, moderate intensity activity is 4.0 METS, and vigorous intensity activity is 8.0 METS (Craig et al., 2003). The MET-min per week for the three categories was computed using MET level $x$ minutes of activity per day multiplied by number of days engaging in the activity per week. The sum of the three categories was used as an independent variable in this study.

\section{Results}

Removing respondents with incomplete responses resulted in $158(72 \%)$ usable responses. The respondents were on average 23.25 years of age with 84 female respondents (53\%). The findings are reported below.

\section{Body Image Satisfaction}

Respondents' ideal body image score was subtracted from their perceived body image score. Respondents who indicate the same score for perceived and ideal body image are satisfied with their body image. However, a positive difference would suggest that respondents perceived themselves to be larger than the ideal body image 
and conversely, a negative difference may suggest that respondents perceived themselves to be smaller than the ideal body size.

The mean difference between the perceived body image and ideal body image for all respondents was .42 $(\mathrm{SD}=1.08)$. Female respondents, however, reported a significantly higher level of dissatisfaction with their body image $(\mathrm{M}=.70, \mathrm{SD}=.82)$ when compared to male respondents $(\mathrm{M}=.11, \mathrm{SD}=1.24 ; t=3.49, p<.01)$.

Further analysis showed that there were other differences between the genders. The proportion of female respondents preferring a larger body $(8 \%)$ is smaller than the proportion of male respondents preferring a larger body $(32 \%)$. Conversely, the proportion of female respondents preferring a smaller body $(69 \%)$ is larger than the proportion of male respondents preferring a smaller body $(42 \%)$. This is illustrated in Figure 1. A ChiSquare test for independence indicated that this difference between the genders was significant, $\chi^{2}(2$, $\mathrm{n}=158)=16.95, p<.01$, Cramer's V=.33.

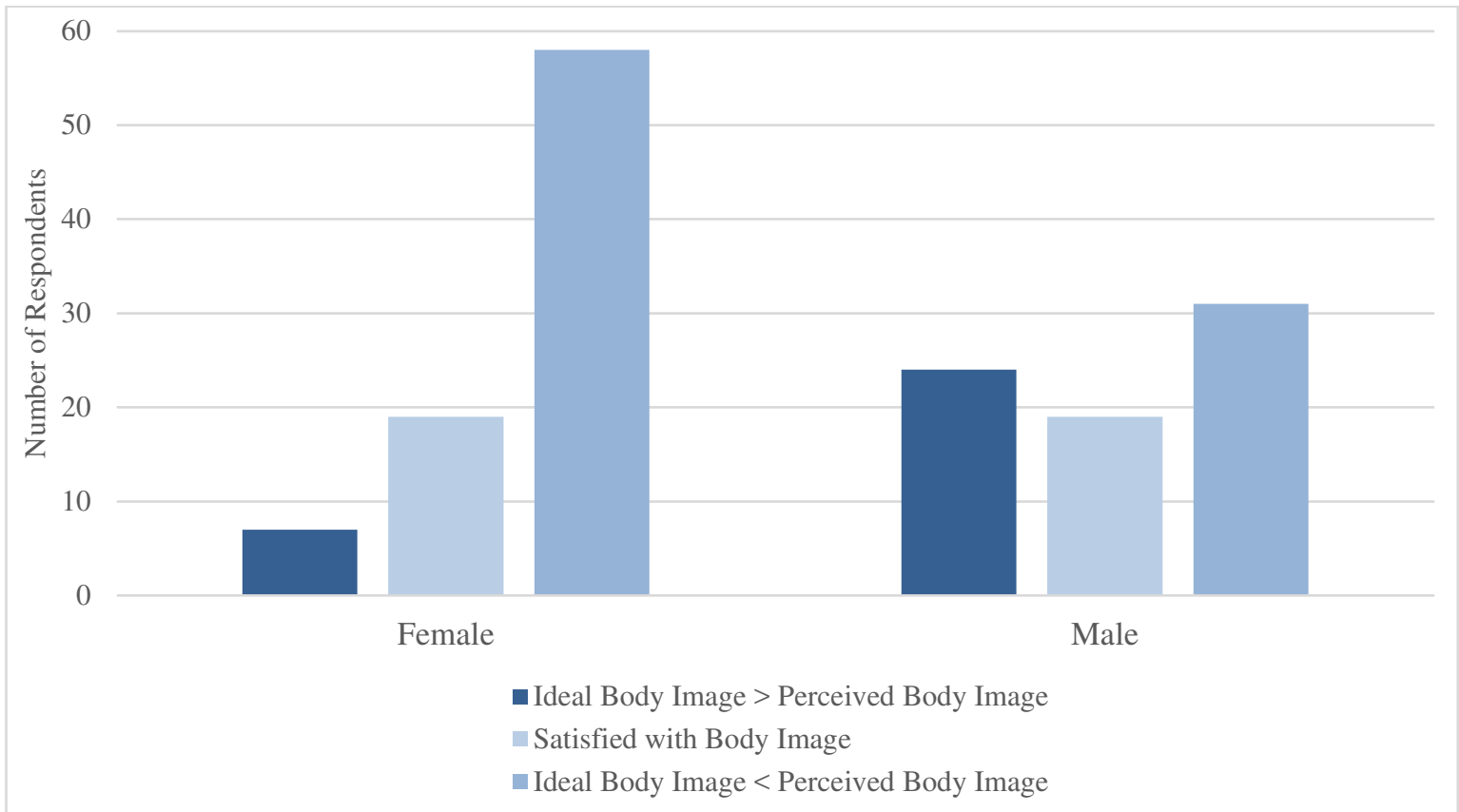

Figure 1 Body Image Satisfaction across Genders

Source: own study

\section{Physical Activity}

Respondents reported a mean MET score of 2444.53 ( $\mathrm{SD}=1706.54$ ). An independent samples t-test was conducted to compare for differences in MET scores across genders. Female respondents reported a significantly lower MET score $(\mathrm{M}=2192.54, \mathrm{SD}=1636.28)$ when compared to male respondents $(\mathrm{M}=2730.57$, $\mathrm{SD}=1750.20 ; t=1.99, p<.05)$. This suggests that female respondents engaged in lower levels of physical activity when compared to male respondents.

The relationship between physical activity (as measured by MET scores) and body image satisfaction (as measured by the difference between ideal and perceived body image) was investigated using the Pearson product-moment correlation coefficient. There was no significant correlation between physical activity and body image satisfaction ( $\mathrm{r}=-.12, \mathrm{n}=84, p>.05)$ for females respondents. Similarly, correlation between physical activity and body image satisfaction was also not significant $(\mathrm{r}=-.18, \mathrm{n}=74, p>.05)$ for male respondents. This suggests that there was no relationship between physical activity and body image satisfaction. 


\section{Sport Involvement}

Respondents reported a mean sport involvement score of 4.60 ( $\mathrm{SD}=1.33$ ). An independent samples t-test showed that female respondents reported a significantly lower sport involvement score $(\mathrm{M}=4.22, \mathrm{SD}=1.31)$ when compared to male respondents $(\mathrm{M}=5.03, \mathrm{SD}=1.24 ; \mathrm{t}=4.00, p<.01)$. The relationship between sport involvement and physical activity was investigated using the Pearson product-moment correlation coefficient. As expected, there was a medium, positive correlation between the two variables for both female $(r=.37, \mathrm{n}=84, p<.01)$ and male $(r=.37, \mathrm{n}=74, p<.01)$ respondents. Respondents who reported a higher level of sport involvement are more likely to be engaged in higher levels of physical activity.

Independent t-tests were conducted to compare for differences in body dissatisfaction between respondents of varying levels of sport involvement. Female respondents who were high in sport involvement, i.e. with a mean sport involvement score higher than 4.00, reported a significantly lower level of dissatisfaction with their body image $(\mathrm{M}=.55, \mathrm{SD}=.78)$ when compared to female respondents who were low in sport involvement $(\mathrm{M}=.84$, $\mathrm{SD}=.83 ; t=1.65, p=.05)$. There was no significant difference amongst male respondents. This is detailed in Table 1 below.

Table 1 Differences in Body Image Satisfaction between high and low sport involvement groups

\begin{tabular}{lcccc}
\hline & $\begin{array}{l}\text { Sport Involvement } \\
\text { Scores }>\mathbf{4 . 0 0}\end{array}$ & $\begin{array}{l}\text { Sport Involvement } \\
\text { Scores }<=\mathbf{4 . 0 0}\end{array}$ & t-statistic & $\begin{array}{c}\text { Eta } \\
\text { squared }\end{array}$ \\
\hline Female & $.55(\mathrm{SD}=.78)^{\mathbf{a}}$ & $.84(\mathrm{SD}=.83)^{\mathbf{b}}$ & $1.65^{*}$ & .03 \\
Male & $.07(\mathrm{SD}=1.22)^{\mathrm{c}}$ & $.22(\mathrm{SD}=1.35)^{\mathrm{d}}$ & .42 & .00 \\
\hline
\end{tabular}

\section{Source: own study}

Note. ${ }^{a} n=40 .{ }^{\mathrm{b}} n=44 .{ }^{\mathrm{c}} n=56 .{ }^{\mathrm{b}} n=18$.

$* p=.05$

The findings suggest that there is a need to examine the relationship between sport involvement and body image among female respondents further. An independent samples t-test showed that female respondents who were high in sport involvement reported a significantly lower score on perceived body image $(\mathrm{M}=3.85, \mathrm{SD}=.92)$ when compared to female respondents who were low in sport involvement $(\mathrm{M}=4.23, \mathrm{SD}=1.01 ; t=1.79, p<.05)$.

Independent sample t-tests were also conducted on ideal body image scores amongst female respondents. Female respondents who were high in sport involvement reported an ideal body image score $(\mathrm{M}=3.30, \mathrm{SD}=.65)$ that is lower when compared to female respondent who were low in sport involvement $(\mathrm{M}=3.39, \mathrm{SD}=.62)$. However, this did not reach statistical significance ( $t=.62, p>.05)$. This is detailed in Table 2 below.

Table 2 Differences in Perceived and Ideal Body Image amongst females with varying levels of sport involvement

\begin{tabular}{lcccc}
\hline & $\begin{array}{l}\text { Sport Involvement } \\
\text { Scores } \boldsymbol{> 4 . 0 0}^{\mathbf{a}}\end{array}$ & $\begin{array}{l}\text { Sport Involvement } \\
\text { Scores }<=\mathbf{4 . 0 0}^{\mathbf{b}}\end{array}$ & t-statistic & Eta squared \\
\hline $\begin{array}{l}\text { Perceived } \\
\text { Body Image }\end{array}$ & $3.85(\mathrm{SD}=.92)$ & $4.23(\mathrm{SD}=1.01)$ & $1.79^{*}$ & .04 \\
$\begin{array}{l}\text { Ideal Body } \\
\text { Image }\end{array}$ & $3.30(\mathrm{SD}=.65)$ & $3.39(\mathrm{SD}=.62)$ & .62 & .00 \\
\hline
\end{tabular}

Source: own study

Note. ${ }^{a} n=40 .{ }^{\mathrm{b}} n=44$.

$* p<.05$

The above shows that female respondents who were high in sport involvement indicated a smaller body size when compared to female respondents who were low in sport involvement. However, there is no difference in the ideal body image between the two groups based on difference in sport involvement. 


\section{Conclusion}

The findings from this study showed that there were differences between the genders in body image satisfaction. Female respondents were more likely to be dissatisfied with their bodies, generally preferring a smaller body size. Conversely, male respondents who are dissatisfied with their bodies may prefer either a larger or small body. This concurs with the literature on gender difference in body image satisfaction (Cohane \& Pope Jr, 2001; Gaspar et al., 2011; Ingledew \& Sullivan, 2002; Smith et al., 2006).

The study did not find any significant relationship between the level of physical activity and body image satisfaction. Earlier studies have suggested that engaging in exercises or physical activity can reduce dissatisfaction with body image. However, some studies have suggested that there were other factors moderating the relationship including environmental influences and motivation of individuals. Consequently, the findings from this study concurred with the existing literature that the relationship between physical activity and body image satisfaction was complex (Prichard \& Tiggemann, 2008). It is not sufficient to encourage individuals who are dissatisfied with their body image to engage in higher levels of physical activity as this does not reduce the level of dissatisfaction with body image.

This study, perhaps more importantly, suggests that the answer to reducing body image dissatisfaction may lie in the cognitive domain eg sport involvement. While sport involvement is positively correlated with physical activity, they are not the same. Sport involvement is a psychological construct and describes an individual's interest and identification with sports (Gwinner \& Swanson, 2003). It does not necessarily involve physical activity as it includes passive activities like watching sports events. Physical activity, on the other hand, is a behavioural construct. It does not dictate whether an individual derives pleasurable emotions in engaging in the activity but merely describes the frequency and duration of activity.

In this study, female respondents who were high in sport involvement were less dissatisfied with their body image. While there is no significant difference among female respondents of varying levels of sport involvement in their ideal body image, female respondents who were high in sport involvement perceived themselves to be smaller in size. While it is beyond the scope of the study and remains to be tested empirically, it suggests that perhaps when female respondents are interested in sport, they may be exposed to different body sizes and become more comfortable with their own bodies. Consequently, they may perceive themselves to be closer to the ideal body image. This follows the findings from earlier studies which suggests that an acceptable body image can be created through socialisation (Baker et al., 2019; Swami et al., 2009; Thompson et al., 1999; Thompson \& Stice, 2001).

In particular, when compared to the findings on the relationship between physical activity and satisfaction with body image, it further suggests that it is not the behaviour of exercising but the psychological aspects of being involved in sport that can affect satisfaction with body image. This suggests that strategies to reduce body image dissatisfaction could be targeted at the cognitive rather than at the behavioural level.

Interestingly, a relationship between sport involvement and body image satisfaction was not found for male respondents in this study. This perhaps stems from the differences between the genders in their preference of an ideal body size. Males can either prefer a larger or smaller body size but females generally prefer a smaller body size. Consequently, this area needs to be examined further.

\section{Ethics approval and informed consent}

Ethical approval for this study was obtained via the school's institutional review board (IRB-2017-02-005).

\section{Competing interests}

There is no conflicting interest of note from any of the authors in the conduct of this research. 


\section{Funding}

There is no funding associated with this study.

\section{REFERENCES}

Abbott, B.D., \& Barber, B.L. (2011). Differences in functional and aesthetic body image between sedentary girls and girls involved in sports and physical activity: Does sport type make a difference? Psychology of Sport and Exercise, 12(3), 333342.

Altabe, M. (1998). Ethnicity and body image: Quantitative and qualitative analysis. International Journal of Eating Disorders, 23(2), 153-159.

Anton, S.D., Perri, M.G., \& Riley III, J.R. (2000). Discrepancy between actual and ideal body images: Impact on eating and exercise behaviors. Eating Behaviors, 1(2), 153-160.

Baker, N., Ferszt, G., \& Breines, J.G. (2019). A Qualitative Study Exploring Female College Students' Instagram Use and Body Image. Cyberpsychology, Behavior, and Social Networking, 22(4), 277-282.

Campbell, A., \& Hausenblas, H.A. (2009). Effects of exercise interventions on body image: A meta-analysis. Journal of Health Psychology, 14(6), 780-793.

Carmona, J., Tornero-Quinones, I., \& Sierra-Robles, Á. (2015). Body image avoidance behaviors in adolescence: A multilevel analysis of contextual effects associated with the physical education class. Psychology of Sport and Exercise, 16, 70-78.

Černelič-Bizjak, M., \& Jenko-Pražnikar, Z. (2014). Impact of negative cognitions about body image on inflammatory status in relation to health. Psychology \& Health, 29(3), 264-278.

Chew, S.S., \& Leng, H.K. (2014). Marketing sports products on Facebook: the effect of social influence. Physical Culture and Sport. Studies and Research, 61(1), 65-73.

Cohane, G.H., \& Pope Jr, H.G. (2001). Body image in boys: A review of the literature. International Journal of Eating Disorders, 29(4), 373-379.

Craig, C.L., Marshall, A.L., Sjorstrom, M., Bauman, A.E., Booth, M.L., Ainsworth, B.E.,... Sallis, J.F. (2003). International physical activity questionnaire: 12-country reliability and validity. Medicine and Science in Sports and Exercise, 35(8), 1381-1395.

Diedrichs, P.C., \& Lee, C. (2011). Waif goodbye! Average-size female models promote positive body image and appeal to consumers. Psychology \& Health, 26(10), 1273-1291.

Fisher, E., \& Thompson, J. K. (1994). A comparative evaluation of cognitive-behavioral therapy (CBT) versus exercise therapy (ET) for the treatment of body image disturbance: Preliminary findings. Behavior Modification, 18(2), 171-185.

Gardner, R.M., Stark, K., Jackson, N.A., \& Friedman, B.N. (1999). Development and validation of two new scales for assessment of body-image. Perceptual and Motor Skills, 89(3), 981-993.

Gaspar, M.J.M., Amaral, T.F., Oliveira, B.M., \& Borges, N. (2011). Protective effect of physical activity on dissatisfaction with body image in children-A cross-sectional study. Psychology of Sport and Exercise, 12(5), 563-569.

Gonçalves, S.F., \& Gomes, A.R. (2012). Exercising for weight and shape reasons vs. health control reasons: The impact on eating disturbance and psychological functioning. Eating Behaviors, 13(2), 127-130.

Graham, M.A., Eich, C., Kephart, B., \& Peterson, D. (2000). Relationship among body image, sex, and popularity of high school students. Perceptual and motor skills, 90(3_suppl), 1187-1193.

Griffiths, S., Murray, S.B., Krug, I., \& McLean, S.A. (2018). The contribution of social media to body dissatisfaction, eating disorder symptoms, and anabolic steroid use among sexual minority men. Cyberpsychology, Behavior, and Social Networking, 21(3), 149-156.

Grogan, S. (2006). Body image and health: contemporary perspectives. Journal of health psychology, 11(4), 523-530.

Gwinner, K., \& Swanson, S.R. (2003). A model of fan identification: Antecedents and sponsorship outcomes. Journal of Services Marketing, 17(3), 275-294.

Hausenblas, H.A., \& Downs, D.S. (2001). Comparison of body image between athletes and nonathletes: A meta-analytic review. Journal of Applied Sport Psychology, 13(3), 323-339.

Hausenblas, H.A., \& Fallon, E.A. (2006). Exercise and body image: A meta-analysis. Psychology \& health, 21(1), 33-47.

Haworth-Hoeppner, S. (2000). The critical shapes of body image: The role of culture and family in the production of eating disorders. Journal of Marriage and Family, 62(1), 212-227. 
Homan, K.J., \& Tylka, T.L. (2014). Appearance-based exercise motivation moderates the relationship between exercise frequency and positive body image. Body Image, 11(2), 101-108.

Hulley, A.J., \& Hill, A.J. (2001). Eating disorders and health in elite women distance runners. International Journal Of Eating Disorders, 30(3), 312-317.

Ingledew, D.K., \& Sullivan, G. (2002). Effects of body mass and body image on exercise motives in adolescence. Psychology of Sport and Exercise, 3(4), 323-338.

Kantanista, A., Glapa, A., Banio, A., Firek, W., Ingarden, A., Malchrowicz-Mośko, E., ... Maćkowiak, Z. (2018). Body image of highly trained female athletes engaged in different types of sport. BioMed Research International, 2018.

Leng, H.K., Kuo, T.Y., Baysa-Pee, G., \& Tay, J. (2014). Make me proud! Singapore 2010 Youth Olympic Games and its effect on national pride of young Singaporeans. International Review for the Sociology of Sport, 49(6), 745-760.

Lewallen, J., \& Behm-Morawitz, E. (2016). Pinterest or thinterest?: Social comparison and body image on social media. Social Media + Society, 2(1), 2056305116640559.

MacKinnon, D.P., Goldberg, L., Cheong, J., Elliot, D., Clarke, G., \& Moe, E. (2003). Male body esteem and physical measurements: Do leaner, or stronger, high school football players have a more positive body image? Journal of Sport and Exercise Psychology, 25(3), 307-322.

Meng, J., Bissell, K.L., \& Pan, P.-L. (2015). YouTube Video as Health Literacy Tool: A Test of Body Image Campaign Effectiveness. Health Marketing Quarterly, 32(4), 350-366.

Nerini, A. (2015). Media influence and body dissatisfaction in preadolescent ballet dancers and non-physically active girls. Psychology of Sport and Exercise, 20, 76-83.

Nustad, J., Adams, T., \& Moore, M. (2008). Health information sources accessed by college females: Differences between body-image distorted and non-body-image distorted. Health Marketing Quarterly, 25(3), 241-253.

Perelman, H., Buscemi, J., Dougherty, E., \& Haedt-Matt, A. (2018). Body Dissatisfaction in Collegiate Athletes: Differences Between Sex, Sport Type, and Division Level. Journal of Clinical Sport Psychology, 12(4), 718-731.

Pila, E., Barlow, M.A., Wrosch, C., \& Sabiston, C.M. (2016). Comparing the body to superior others: Associations with daily exercise and body evaluation in men and women. Psychology of Sport and Exercise, 27, 120-127.

Posavac, H.D., Posavac, S.S., \& Weigel, R.G. (2001). Reducing the impact of media images on women at risk for body image disturbance: Three targeted interventions. Journal of Social and Clinical Psychology, 20(3), 324-340.

Prichard, I., \& Tiggemann, M. (2008). Relations among exercise type, self-objectification, and body image in the fitness centre environment: The role of reasons for exercise. Psychology of Sport and Exercise, 9(6), 855-866.

Raedeke, T.D. (2007). The relationship between enjoyment and affective responses to exercise. Journal of Applied Sport Psychology, 19(1), 105-115.

SantaBarbara, N.J., Whitworth, J.W., \& Ciccolo, J.T. (2017). A systematic review of the effects of resistance training on body image. The Journal of Strength \& Conditioning Research, 31(10), 2880-2888.

Smith, D., Wright, C., Ross, N., \& Warmington, S. (2006). Sports Advertising and Body Image Body Image: New Research (pp. 65-73): Nova Science Publisher.

Strelan, P., Mehaffey, S.J., \& Tiggemann, M. (2003). Brief report: Self-objectification and esteem in young women: The mediating role of reasons for exercise. Sex Roles, 48(1-2), 89-95.

Stunkard, A. (2000). Old and new scales for the assessment of body image. Perceptual and Motor Skills, 90(3), 930-930.

Swami, V., Steadman, L., \& Tovée, M.J. (2009). A comparison of body size ideals, body dissatisfaction, and media influence between female track athletes, martial artists, and non-athletes. Psychology of Sport and Exercise, 10(6), 609614.

Thompson, J.K., Heinberg, L.J., Altabe, M., \& Tantleff-Dunn, S. (1999). Exacting beauty: Theory, assessment, and treatment of body image disturbance: American Psychological Association.

Thompson, J.K., \& Stice, E. (2001). Thin-ideal internalization: Mounting evidence for a new risk factor for body-image disturbance and eating pathology. Current Directions in Psychological Science, 10(5), 181-183.

Vocks, S., Hechler, T., Rohrig, S., \& Legenbauer, T. (2009). Effects of a physical exercise session on state body image: The influence of pre-experimental body dissatisfaction and concerns about weight and shape. Psychology \& Health, 24(6), 713-728.

Wardle, J., Bindra, R., Fairclough, B., \& Westcombe, A. (1993). Culture and body image: Body perception and weight concern in young Asian and Caucasian British women. Journal of Community \& Applied Social Psychology, 3(3), 173181. 
You, S., \& Shin, K. (2016). Body dissatisfaction and mental health outcomes among Korean college students. Psychological reports, 118(3), 714-724.

\section{AUTHOR'S ADDRESS:}

Ho Keat Leng

National Institute of Education

Nanyang Technological University

1 Nanyang Walk

Singapore 637616

E-mail: hokeat.leng@nie.edu.sg

Received: 18 July 2019; Accepted: 23 October 2019 